\title{
ESTRATÉGIAS PARA O ESTUDO DE DINÂMICA DE EIXOS ROTATIVOS NO PERÍODO DE ENSINO REMOTO
}

DOI: 10.37702/2175-957X.COBENGE.2021.3753

Fernando da Silva Araújo - fernando.araujo@cefet-rj.br

CEFET RJ

Rua do Areal 522

23953-030 - Angra dos Reis - RJ

Resumo: Este trabalho tem por objetivo mostrar estratégias de ensino para o estudo de dinâmica de eixos rotativos, na disciplina Dinâmica das Máquinas que compõe o ciclo profissionalizante de disciplinas da Graduação em Engenharia Mecânica, do CEFET/RJ Campus Angra dos Reis no período de ensino remoto emergencial. Este tipo de metodologia ativa de aprendizagem proporciona motivação, interesse, autonomia e interação dos alunos diante de cada uma das etapas realizadas no trabalho. $O$ projeto proposto no período de ensino remoto foi a determinação da frequência natural e deslocamentos de um eixo rotativo composto de rotores (massas) por meio de método analítico e simulação computacional usando o software Inventor. Em seguida, os alunos elaboraram um relatório do trabalho em formato de artigo científico. Para a execução do trabalho, formou-se grupos de dois ou três alunos a fim de estimular o trabalho em equipe. Além disso, aplicou-se um questionário sobre os aspectos metodológicos da atividade no ensino remoto. A partir das respostas ao questionário foi verificada a importância da estratégia de ensino empregada com a qual os alunos conseguiram assimilar o assunto e compreenderam fenômenos complexos de forma mais fácil e motivadora.

Palavras-chave: Aprendizagem ativa. Ensino de engenharia mecânica. Rodopio de eixo. 


\section{INTRODUÇÃO}

Segundo as novas DCNs de Engenharia, uma das competências que o egresso deve apresentar é ser capaz de modelar os fenômenos, os sistemas físicos e químicos, utilizando as ferramentas matemáticas, estatísticas, computacionais e de simulação, entre outras. $O$ uso de ferramenta computacional vem sendo bastante utilizada no processo de ensino aprendizagem de engenharia (CARVALHO et al., 2020; SILVA et.al, 2020).

A análise modal é o processo constituído de técnicas teóricas e experimentais que possibilitam a elaboração de um modelo matemático que represente o comportamento dinâmico do sistema em estudo, a fim de determinar os seus parâmetros modais: modos de vibração, fatores de amortecimento e frequências naturais (NÓBREGA e HANAI, 2005).

Determinar a frequência natural de um sistema é de extrema importância no estudo da engenharia, pois por meio de seu resultado é possível evitar a ocorrência do fenômeno de ressonância, ou seja, quando a frequência de operação de uma máquina for igual a sua frequência natural, podendo levar um equipamento ou estrutura a desgastes excessivos e falhas (BOLINA et al., 2015). Para determinar a frequência natural de um sistema pode-se adotar métodos analíticos, experimentais e numéricos. Daí, a relevância de um estudo criterioso desse fenômeno para a elaboração de projeto de máquinas e estruturas confiável.

Por sua vez, nesse período de ensino remoto devido à pandemia de Covid-19, os professores precisaram se reinventar para conseguir lecionar e, assim, atingir o objetivo de fazer com que o aluno estude e aprenda com motivação. O professor Jim Kurose (KUROSE, 2021), da Universidade de Massachusets Amherst em aula inaugural da Coppe/UFRJ, acredita que algumas mudanças na forma de ensinar e de aprender vieram para ficar, e que o maior desafio será garantir a equidade digital, um acesso justo e igualitário ao conhecimento nesta modalidade de ensino virtual. Ainda segundo o referido professor, surge uma nova pedagogia em que há uma mudança de ensino expositivo para aprendizado ativo (active learning) com mais interação.

$\mathrm{O}$ ensino de engenharia por se só gera desafios ainda maiores devido à sua complexidade e peculiaridade no que tange os fenômenos físicos associados. Além disso, deve-se levar em consideração que laboratórios para estudos e análises experimentais são onerosos e, neste momento inacessíveis, mas, por meio de ferramentas computacionais, é possível, calcular e identificar parâmetros dinâmicos de sistemas (SILVA, 2018).

Diante do exposto, este trabalho tem por objetivo desenvolver uma estratégia de ensino para o estudo de dinâmica de eixos rotativos utilizando simulação computacional e cálculo analítico na determinação de frequências naturais, modos de vibração e deflexões de um eixo que suporta três rotores.

\section{MODELAGEM MATEMÁTICA}

O cálculo da frequência natural de sistemas mecânicos pode ser feito por meio de diversos métodos analíticos. É possível destacar entre esses o método de Rayleigh, a fórmula de Dunkerley e o método de Holzer. A escolha do método depende do problema proposto (Rao, 2008; Uicker et al., 2017). Neste trabalho, objetivou-se estimar a frequência fundamental de vibração transversal de um eixo que porta três rotores (massas), conforme apresentado na Figura 1.

O método de Rayleigh assume que a curva de deflexão estática é usada como uma aproximação da curva de deflexão dinâmica. Assim, a energia máxima potencial do sistema 
devida à energia de deformação do eixo defletido é igual a energia cinética máxima devida à velocidade e às massas. Então, a primeira frequência fundamental do eixo é dada por

$$
\omega=\left\{\frac{g\left(m_{1} w_{1}+m_{2} w_{2}+\cdots\right.}{\left.m_{1} w_{1}^{2}+m_{2} w_{2}^{2}+\cdots\right)}\right\}^{\frac{1}{2}}
$$

Em que $g$ é a aceleração da gravidade, $m_{i}$ e $w_{i}$ é a massa e a deflexão estática total da massa $m_{i}$ do i-ésimo elemento, respectivamente.

O modelo a ser estudado foi retirado do livro Vibrações Mecânicas (Rao, 2008). O exemplo descreve um eixo feito de aço de formato cilíndrico maciço nas dimensões especificadas de $10 \mathrm{~m}$ de comprimento com um diâmetro de $100 \mathrm{~mm}$, sustentando três rotores dispostos ao longo de seu comprimento com massas: $m_{1}=20 \mathrm{~kg}, m_{2}=50 \mathrm{~kg}$ e $m_{3}=$ $40 \mathrm{~kg}$ nas distancias: $l_{1}=1 \mathrm{~m}, l_{2}=3 \mathrm{~m}, l_{3}=4 \mathrm{~m}$ e $l_{4}=2 \mathrm{~m}$ e módulo de elasticidade do aço de $E=207 \times 10^{9} \mathrm{~N} / \mathrm{mm}^{2}$.

Figura 1 - Eixo suportando três rotores (massas).

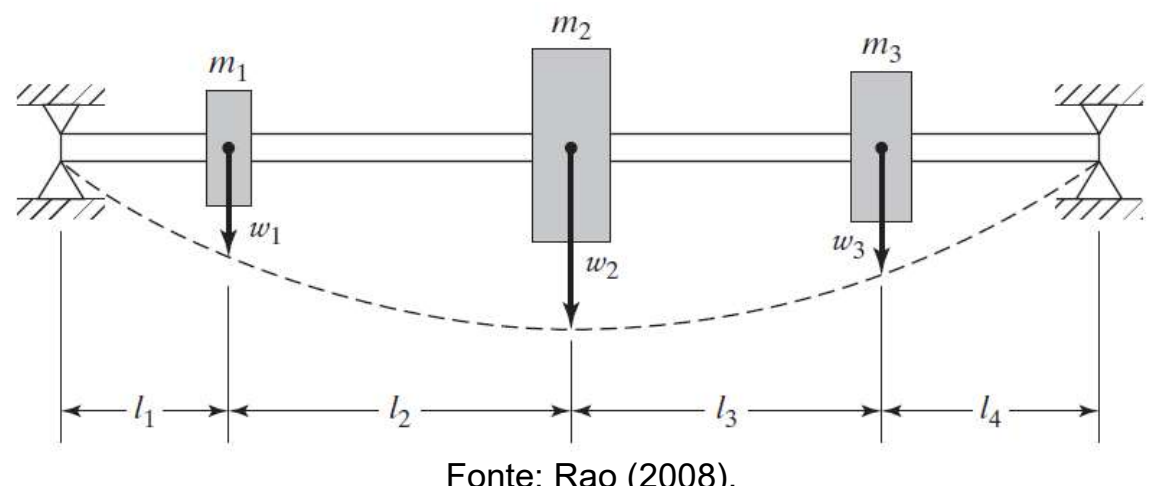

Pela teoria de resistência dos materiais, a deflexão em função da distância $x$ do primeiro apoio de uma viga de comprimento $l$, bi-apoiada, com uma carga estática $P$ aplicada a uma distância $a$ e $b$ dos apoios é dada pela Equação (2).

$$
w(x)=\left\{\begin{array}{c}
\frac{P b x}{6 E I l}\left(l^{2}-b^{2}-x^{2}\right) ; 0 \leq x \leq a \\
-\frac{P a(l-x)}{6 E I l}\left[a^{2}+x^{2}-2 l x\right] ; a \leq x \leq l
\end{array}\right.
$$

Assim, as deflexões totais das massas $m_{1}, m_{2}$ e $m_{3}$ serão dadas pela soma das contribuições de cada massa para a deflexão em cada ponto.

\section{SIMULAÇÃO}

A fim de determinar as frequências naturais e modos de vibração do sistema via método dos elementos finitos (MEF), uma simulação foi realizada considerando a mesma geometria do eixo e carregamentos informados anteriormente. Para simplificação, foram adotadas 3 cargas pontuais representando os pesos dos rotores no eixo para a realização 
da simulação, em que $F_{1}=196,2 N, F_{2}=490,5 N, F_{3}=392,4 N$. A Figura 2 mostra o modelo 3D.

Figura 2 - Modelo do eixo com os pesos dos três rotores na simulação.

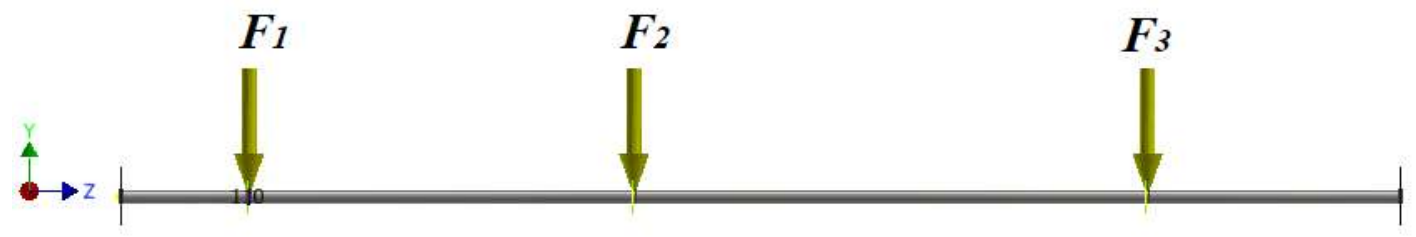

Fonte: Autoria própria.

A simulação foi executada no software Autodesk Inventor 2019, disponibilizado para os alunos do CEFET/RJ. Foram utilizados os módulos de análise estática para determinar as deflexões do eixo quando submetido ao carregamento estático e o de análise modal para determinar as frequências naturais, modos de vibração e deslocamentos do eixo na ressonância. Adotou-se as seguintes configurações para a malha: tamanho médio dos elementos (fração do diâmetro do model) igual a 0,005, tamanho mínimo dos elementos (fração do tamanho médio) igual a 0,01, fator de nivelamento igual a 1 e ângulo máximo de giro igual a $60^{\circ}$.

Selecionou-se no Inventor o material do eixo sendo o aço com massa específica de $7,85 \mathrm{~g} / \mathrm{cm}^{3}$ e módulo de Young igual a $220 \mathrm{GPa}$. O referido software só permite adotar a restrição de apoio do tipo engaste ao invés de pinada nas extremidades do eixo.

\section{APLICAÇÃO DE QUESTIONÁRIO}

Foi elaborado um questionário para os alunos cujo objetivo foi verificar se a estratégia de ensino facilitou o processo de ensino aprendizagem sobre dinâmica de eixos rotativos no período de ensino remoto. As aulas síncronas e assíncronas foram ministradas por meio da plataforma Teams da Microsoft disponibiliza pela instituição. Primeiramente, antes do início do trabalho, a turma foi consultada acerca da acessibilidade e do nível de conhecimento de ferramentas computacionais necessárias para execução do trabalho. Sabe-se que após o término da atividade, o aluno consegue comparar com clareza seu nível de conhecimento antes e depois da realização do trabalho. Então, as perguntas do restante do questionário versaram sobre os aspectos positivos e negativos da metodologia do ensino empregada no trabalho. O formulário foi elaborado na própria plataforma Teams por meio da ferramenta Microsoft forms e disponibilizado por meio de link no ambiente de postagens da turma.

\section{RESULTADOS E DISCUSSÃO}

Primeiramente, foi feito o cálculo analítico dos deslocamentos das massas dos rotores considerando o eixo bi-apoiado. Da Equação (2), o valor do deslocamento total da massa 1, massa 2 e massa 3 foram $4,78 \mathrm{~mm}, 14,6 \mathrm{~mm}$ e 9,05 mm, respectivamente. Em seguida, o valor da frequência natural calculado pela Equação (1) foi de $4,53 \mathrm{~Hz}(28,45$ $\mathrm{rad} / \mathrm{s})$. A simulação no Inventor foi executada, conforme o procedimento descrito no item 3 . Vale destacar que nesta versão para estudante do software só é possível colocar a restrição 
de apoio do tipo engaste no eixo. Assim, o valor numérico da primeira frequência natural do eixo foi de $4,43 \mathrm{~Hz}$. Obteve-se também pela simulação o máximo deslocamento do eixo de $3,2 \mathrm{~mm}$, conforme mostrado na Figura 3. Percebe-se que esta discrepância nos valores da primeira frequência natural e dos deslocamentos calculados por ambos os métodos é devida ao diferente tipo de apoio do eixo, como esperado.

Para fins de comparação, calculou-se de forma analítica o deslocamento da massa 2 utilizando a função de singularidade para o caso do eixo bi-engastado. Então, obteve-se um deslocamento de $2,9 \mathrm{~mm}$ para a massa 2. Desta forma, este valor está condizente com o valor máximo de deslocamento do eixo de $3,18 \mathrm{~mm}$ calculado pelo software. Vale ressaltar que não foi considerado o peso próprio do eixo em nenhuma das análises.

Figura 3 - Deslocamento na direção direção-y para o caso de carregamento estático e eixo bi-engastado.

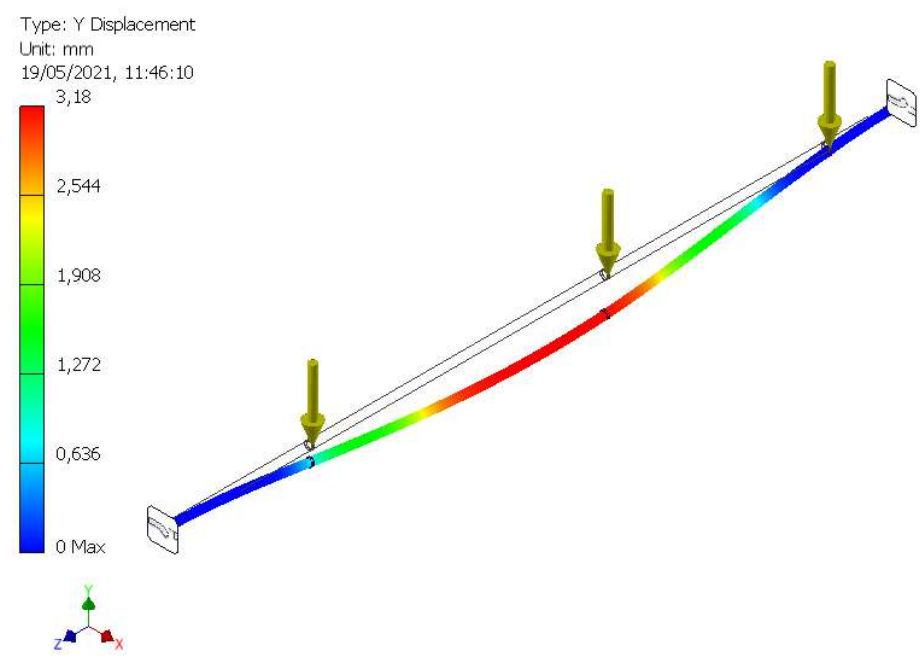

Fonte: Autoria própria.

Foi possível também obter por meio da modelagem numérica, os valores dos deslocamentos quando ocorre o rodopio (whirling) do eixo, isto é, na primeira frequência ressonante ou velocidade crítica de rotação de $4,43 \mathrm{~Hz}$, conforme mostrado na Figura 4. Verifica-se um valor máximo de deslocamento radial de $500,1 \mathrm{~mm}$ que neste caso que é muito maior (da ordem de 170 vezes) que aquele obtido quando o eixo está defletido apenas devido ao carregamento estático dos rotores.

Por meio da simulação numérica, foram obtidos também os modos de vibração na segunda, terceira e quarta frequências naturais, conforme mostrada na Figura 5. Observase que o segundo, terceiro e quarto modo de vibração possuem um, dois e três nós entre os apoios, respectivamente, conforme previsto pela teoria. 


\section{COBENCE de Educação em Engenharia 2021 da ABENGE

Figura 4 - Deslocamento total de rodopio do eixo na primeira frequência fundamental de $4,43 \mathrm{~Hz}$.

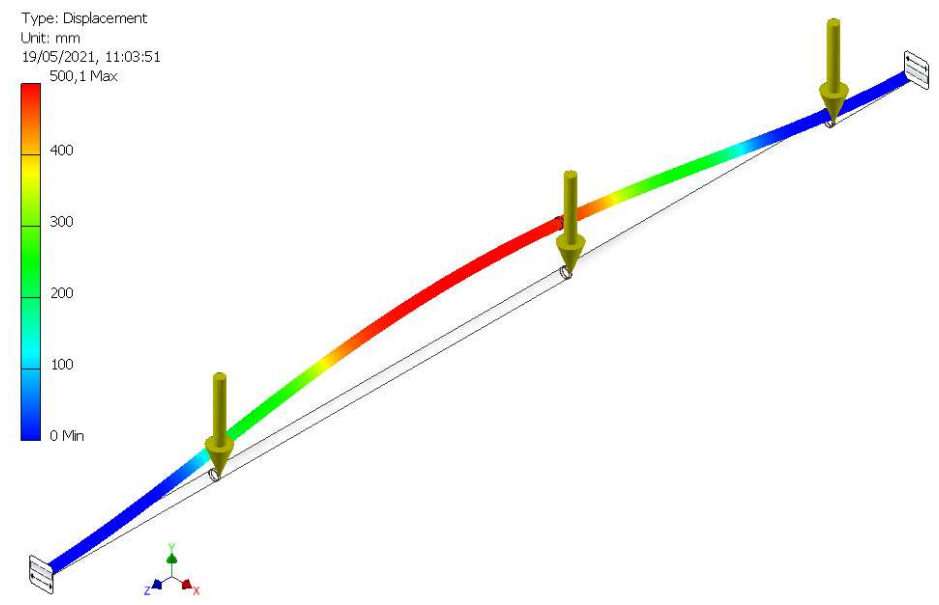

Fonte: Autoria própria.

Figura 5 - Modos de vibração do eixo na segunda frequência natural de $12,17 \mathrm{~Hz}(\mathrm{a})$, terceira frequência natural de $23,91 \mathrm{~Hz}$ (b) e na quarta frequência natural de $39,37 \mathrm{~Hz}$ (c).

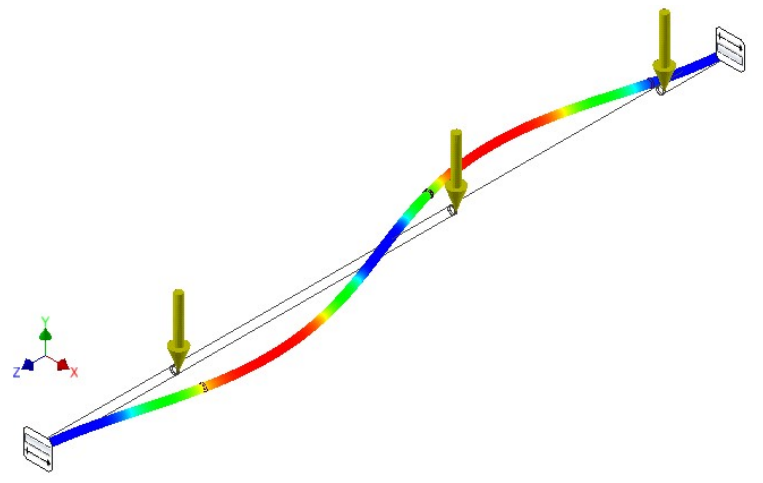

(a)

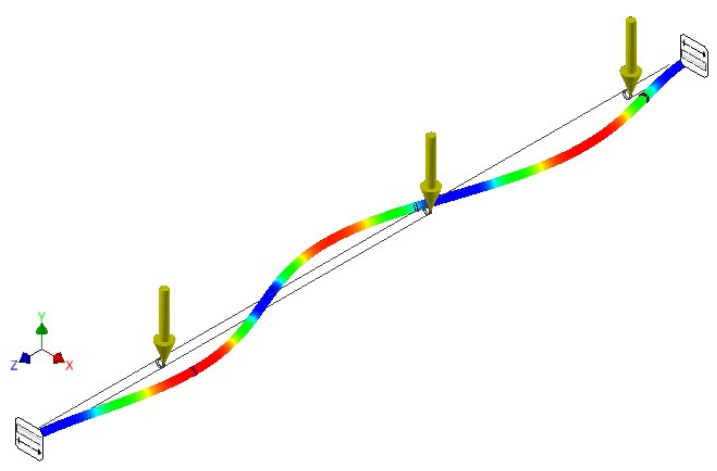

(b)

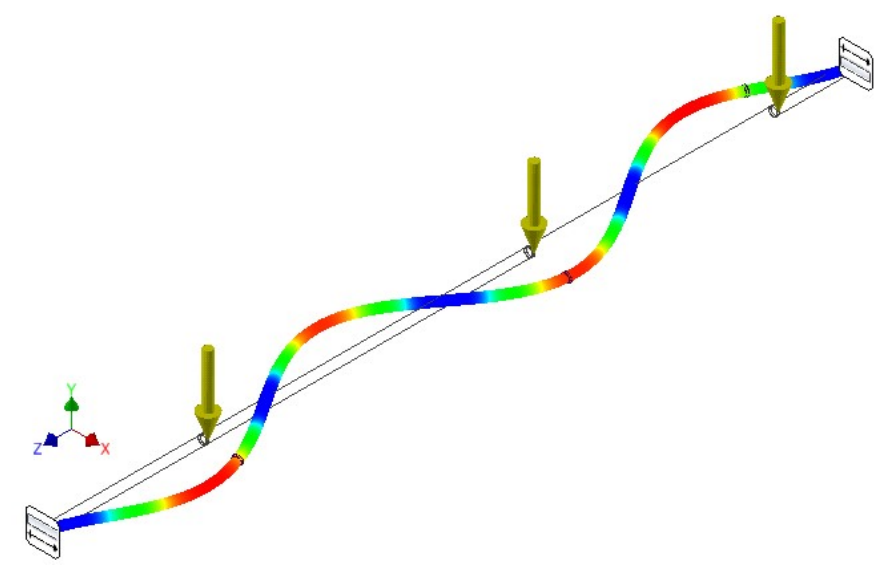

(c)

Fonte: Autoria própria.

Com o objetivo de verificar a percepção dos alunos, foi utilizado um formulário online (Forms office) como estratégia de metodologia ativa na avaliação do trabalho no ensino remoto. Todos os 19 alunos da turma responderam ao questionário cujo tempo médio para concluir foi de sete minutos e meio. A seguir, as respostas ao questionário indicam o 
sucesso da atividade obtido por meio da implementação da estratégia de ensino. Primeiramente, antes do início do trabalho, foram feitas as seguintes perguntas acerca da acessibilidade e do nível de conhecimento dos alunos sobre ferramentas computacionais necessárias para execução do trabalho. $74 \%$ dos alunos não tinham ou tinham habilidades ruins em softwares de simulação e modelagem computacional. Além disso, $84 \%$ da turma não tinha feito um trabalho envolvendo comparação de métodos analíticos e numéricos.

Uma das sugestões apontadas pelos alunos para contornar esta deficiência é a realização de atividades extracurriculares, como minicursos, de modo a introduzir aos alunos o uso de ferramentas de simulação e análise numérica. 39\% relataram que o computador que possuíam não tinha capacidade suficiente para rodar softwares de simulação ou modelagem. Sendo assim, os alunos que não dispunham da ferramenta foram inseridos em grupos de alunos que possuíam computador e software com desempenho satisfatório. Foram disponibilizados para os alunos tutorial do software e vídeos com passo a passo para realização de simulações simples. Os alunos tiveram um tempo para aprender e tirar dúvidas sobre o programa.

$60 \%$ dos alunos não sabiam como acessar e baixar remotamente artigos científicos e livros no Portal de periódicos CAPES/MEC via Comunidade Acadêmica Federada (CAFe) por meio de login e senha do CEFET/RJ. Antes do início do tralhado, foi mostrado para a turma o passo a passo (Figura 6) para acessar este portal pelo site: $h t t p s: / / w w w-p e r i o d i c o s-$ capes-gov-br.ezl.periodicos.capes.gov.br. Vale ressaltar que neste período de ensino remoto, os alunos estão sem acesso à biblioteca da instituição para conseguir livros físicos e material de pesquisa.

Figura 6 - Acesso ao portal de periódicos CAPES/MEC via CAFe: Seleção da instituição de ensino (a); login e senha institucionais do usuário (b)

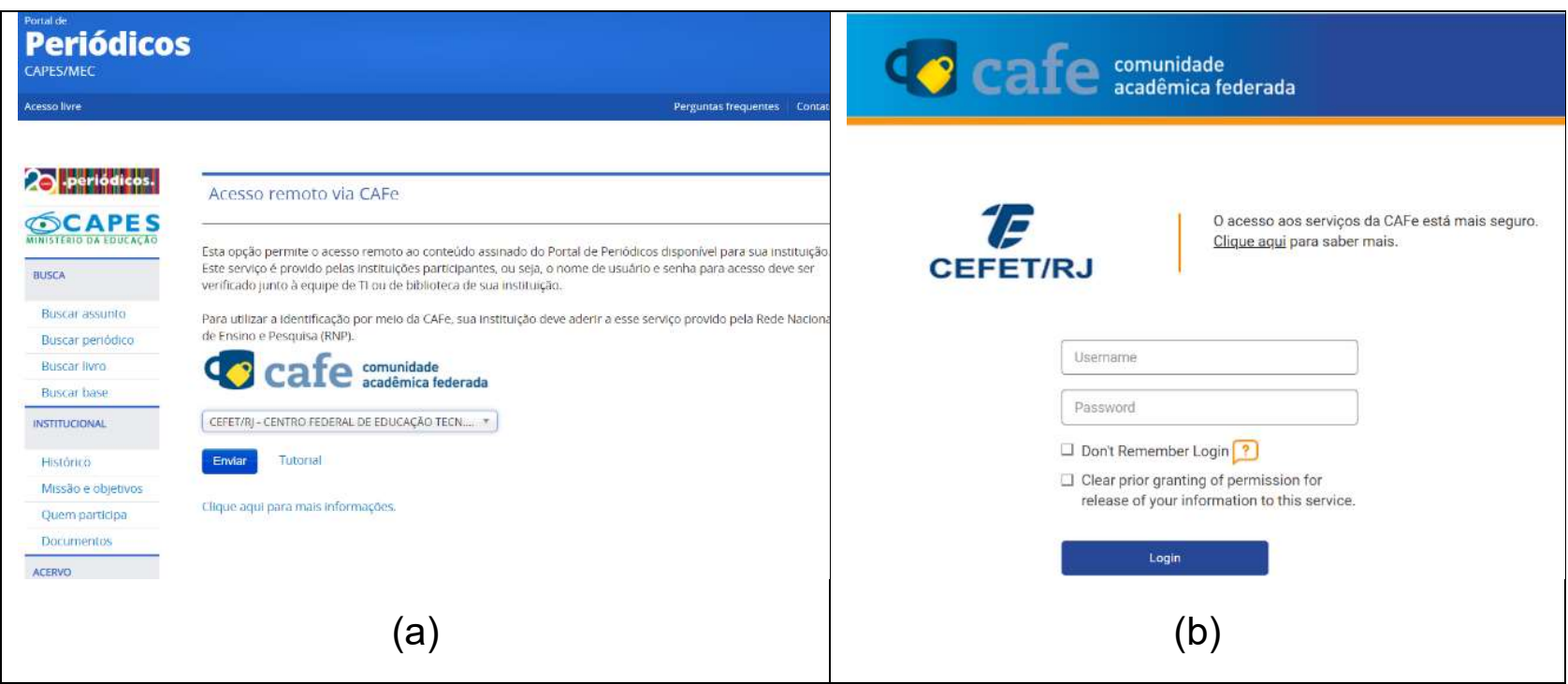

Fonte: Portal de periódicos CAPES/MEC.

Após o término do trabalho, o restante do formulário foi respondido pelos alunos. $90 \%$ dos estudantes afirmam que o uso da simulação ajudou a melhor entender ou visualizar os fenômenos físicos do problema. Todos os alunos apontam que software facilita e otimiza a análise do problema, porém o entendimento dos resultados da simulação ocorre somente quando se conhece previamente a teoria que descreve o fenômeno. Também, 90 $\%$ dos alunos afirmaram que o trabalho ajudou a motivá-los e facilitou o aprendizado do 
conteúdo. Esta percepção mostra o quão importante a metodologia empregada se mostrou atrativa e eficiente no processo de ensino-aprendizagem.

Todos os alunos indicaram que o trabalho ajudou satisfatoriamente a desenvolver outras habilidades e competências como leitura e escrita científica, trabalho em equipe, comunicação, aprendizado de forma autônoma, liderança, planejamento, uso de ferramentas de simulação, etc. Vale ressaltar que os grupos de alunos se reuniam para discutir sobre a execução e andamento do trabalho. A ferramenta Teams permite que os alunos façam vídeo chamada para reunião de equipes ou utilizem o chat em grupo para discussão por mensagens. Os alunos tiveram que entregar a parte escrita do trabalho em formato de artigo científico. Eles foram incentivados a pesquisar artigos em inglês sobre o tema e citá-los no estado da arte do trabalho. Os alunos relataram que esta etapa ajudou no aprofundamento do conhecimento sobre o tema e no aprendizado de diversos termos técnicos em inglês da mecânica utilizados na literatura especializada.

Sobre a questão de esclarecimento de dúvidas e distância entre aluno-professor, $84 \%$ dos alunos não se sentiram mais distante do professor, porém $44 \%$ destes alunos não conseguiram tirar todas as dúvidas acerca do trabalho. Isso reflete a limitação do contato professor-aluno nesta modalidade de ensino. Todos os alunos concordam que a metodologia utilizada deve ser implementada no ensino presencial ou híbrido. Vale destacar que os alunos puderam tirar dúvidas com o professor por meio de "chat", e-mail institucional ou vídeo chamada na plataforma Teams. Há horários específicos para atendimento aos alunos fora do horário de aula. Além disso, os estudantes podem enviar perguntas a qualquer momento via chat e, então, o professor responde-as quando acessa o sistema.

Por fim, os alunos também puderam opinar sobre os desafios, limitações e contribuições do trabalho para melhoria do processo de ensino-aprendizagem. A seguir serão destacados alguns relatos dos alunos.

"O trabalho proporcionou desafios no qual muita das vezes poderemos passar em alguma empresa futuramente" (Aluno A).

"A proximidade aluno-professor é um grande desafio nesse ensino remoto. Experimentos que ajudariam na compreensão da matéria não podem ser realizados nesse tipo de ensino, o que limita um pouco o aprendizado, mas que pode ser contornado por meio de trabalhos envolvendo o uso de softwares" (Aluno B).

"O desenvolvimento do trabalho é bem significativo para o desenvolvimento dos alunos, porém no período remoto a existência de atividades que necessitem de recursos que os alunos não tenham disponíveis levam a uma ansiedade e estresse bem grande" (Aluno C).

"Como contribuições diria a fuga de aprendizados somente teóricos para algo mais prático" (Aluno D).

"Estudos de simulação são essenciais na engenharia e ajuda muito o aluno a entender muitas das coisas que até então estão apenas no conceito" (Aluno E).

"Achei interessante poder olhar o problema com outros olhos e entender como funciona não só no papel. Acho importante termos outros meios de aprendizagem. E é muito legal como podemos ver os fenômenos ocorrendo nas simulações do programa" (Aluno F).

\section{CONSIDERAÇÕES FINAIS}

A partir do projeto proposto na disciplina de Dinâmica das Máquinas no período de ensino remoto, foi possível determinar a frequência natural e amplitudes de vibração de um eixo rotativo composto de rotores (massas) por meio de método analítico e simulação computacional usando o software Inventor. Para a execução do trabalho, formou-se grupos 
de dois ou três alunos a fim de estimular o trabalho em equipe. Além disso, aplicou-se um questionário sobre os aspectos metodológicos da atividade no ensino remoto. A partir das respostas ao questionário foi verificada a importância da estratégia de ensino empregada, por meio do uso de software e simulação, com a qual os alunos participaram ativamente do processo ensino aprendizagem e compreenderam fenômenos complexos de forma mais fácil e motivadora. Estas estratégias podem ser aplicadas para outras disciplinas da área de engenharia.

\section{Agradecimentos}

O autor agradece ao Centro Federal de Educação Tecnológica Celso Suckow da Fonseca (CEFET/RJ), Campus Angra dos Reis, pela estrutura disponibilizada.

\section{REFERÊNCIAS}

BOLINA, C.C.; Palechor, E. U. L.; Vásquez, M. P. R.; Vibrações: As Frequências Naturais Estimada e Experimental de uma Estrutura. In: Congresso Nacional de Matemática Aplicada à Indústria.: Blucher, 2015. Anais. São Paulo. Disponível em:

https://www.proceedings.blucher.com.br/article-details/vibraes-as-frequncias-naturaisestimada-e-experimental-de-uma-estrutura-11900. Acesso em 20 abr.2021.

CARVALHO, A. S.; ALMEIDA, G. C. S.; SALLES, R. S. Aspectos da Utilização de uma Plataforma Tecnológica no Ensino de Pid Tuning. In: XLVIII Congresso Brasileiro de Ensino de Engenharia - COBENGE. Caxias do Sul, RS. Anais. 2020.

KUROSE, Jim. Palestra de aula inaugural. Planeta Coppe notícias. Disponível em: https://coppe.ufrj.br/pt-br/planeta-coppe-noticias/noticias/jim-kurose-defende-aprendizadoativo-e-equidade-digital-na-aula. Acesso em: 10 mai. 2021.

NÓBREGA, P.G.B.; HANAI, J.B. A. Análise Modal na Avaliação de Estruturas de Concreto Pré-moldado. $1^{\circ}$ Encontro Nacional de Pesquisa-Projeto-Produção em Concreto pré-moldado. São Carlos, 2005

RAO, Singiresu S. Vibrações Mecânicas. 4. Ed, Printece Hall Brasil, 2009.

SILVA, A. D. C. et al. Desenvolvimento de uma interface gráfica para uso acadêmico do software opendss. In: XLVIII Congresso Brasileiro de Ensino de Engenharia - COBENGE. Caxias do Sul, RS. Anais. 2020.

\section{SILVA, L. G. ANÁLISE ESTRUTURAL E MODAL DE UM CHASSI DE MOTOCICLETA.} Monografia (Graduação) - UFPB/Tecnologia. João Pessoa, 2018.

Uicker, John Joseph.; Pennock, G. R.; Shigley, J. E. Theory of machines and mechanisms. 5. Ed, New York, Oxford University Press, 2016. 


\title{
STRATEGIES FOR STUDYING DYNAMICS OF ROTATING SHAFTS IN THE REMOTE- TEACHING PERIOD
}

\begin{abstract}
This work aims to show teaching strategies for the study of dynamics of rotating shafts, in the subject of Dynamics of Machinery that make up the professional cycle of disciplines of the Graduation in Mechanical Engineering, of CEFET / RJ Campus Angra dos Reis during the emergency remote teaching. This type of active learning provides motivation, interest, autonomy and interaction of the students in each stage of the work. The proposed activity was the determination of the natural frequency and displacements of a rotating shaft composed of rotors or discs (masses) by means of an analytical method and computational simulation using Inventor. Then, the students prepared a report using a scientific article template. For the execution of the work, groups of two or three students were created in order to encourage teamwork. In addition, an online form was applied to capture the student's perception about the methodological aspects of the activity during the remote teaching. From the answers collected in the form, the importance of the teaching strategy employed was verified in which the students were able to assimilate the subject and understood complex phenomena in an easier and more motivating way.
\end{abstract}

Keywords: Active Learning, Mechanical Engineering Teaching, Whirling of Rotating Shafts 